\title{
Prognostic impact of right ventricular dysfunction following transcatheter mitral valve repair
}

Shingo Kuwata ${ }^{1}$, Masaki Izumo ${ }^{1}$, noriko Shiokawa ${ }^{1}$, Sato Yukio ${ }^{1}$, Ryo Kamijima ${ }^{1}$, Shunichi Doi $^{1}$, Haruka Nishikawa ${ }^{1}$, Toshiki Kaihara ${ }^{1}$, Masashi Koga ${ }^{1}$, Kazuaki Okuyama ${ }^{1}$, Yasuhiro Tanabe $^{1}$, Tomoo Harada ${ }^{1}$, Yuki Ishibashi ${ }^{1}$, and Yoshihiro Akashi ${ }^{1}$

${ }^{1}$ St. Marianna University School of Medicine

July 11, 2021

\begin{abstract}
Background Little is known regarding the impact of right ventricular (RV) function on clinical outcomes following MitraClip therapy. Objectives The aim of this study was to investigate the prognostic impact of RV dysfunction and its cut-off value following MitraClip therapy. Methods Consecutive 77 patients (median 79 years, $33 \%$ female) who underwent MitraClip therapy were enrolled. Clinical endpoint was defined as cardiovascular (CV) events, including cardiovascular death and rehospitalization for heart failure (HF). Results and conclusions Twenty-two (29\%) patients had primary mitral regurgitation (MR). During follow-up, 5 patients died due to CV events, 8 were hospitalized for HF. On univariate Cox regression analysis, CV events were associated with eGFR (HR; 0.960, 95\% CI; 0.926-0.995, p = 0.027), tricuspid annular plane systolic excursion (TAPSE, HR; 0.874, 95\% CI; 0.789-0.968, p = 0.010), and significant residual MR (HR; 11.652, 95\% CI; 3.257-41.691, p <0.001). On multivariate Cox regression analysis, TAPSE (HR; 0.788, 95\% CI; 0.788-0.987, p = 0.029) and significant residual MR (HR; 9.373, 95\% CI; 2.581-34.033, $\mathrm{p}=0.001)$ were independently associated with CV events. TAPSE $<11$ mm was the best cut-off criteria for predicting CV events. RV function was independently associated with clinical outcomes following MitraClip therapy. TAPSE is a simple parameter for predicting CV events in patients with MR who are undergoing MitraClip therapy.
\end{abstract}

Prognostic impact of right ventricular dysfunction following transcatheter mitral valve repair Running title: Prognostic impact of TAPSE on MitraClip therapy

Shingo Kuwata ${ }^{1}$, MD; Masaki Izumo ${ }^{1}$, MD; Noriko Shiokawa ${ }^{2}$, JRDCS; Yukio Sato ${ }^{1}$, MD; Ryo Kamijima ${ }^{1}$, MD; Shunichi Doi ${ }^{1}$, MD; Haruka Kameshima ${ }^{1}$, MD; Toshiki Kaihara ${ }^{1}$, MD; Masashi Koga ${ }^{1}$, MD; Kazuaki Okuyama $^{1}$, MD; Yasuhiro Tanabe ${ }^{1}$, MD; Tomoo Harada ${ }^{1}$, MD; Yuki Ishibashi $^{1}$, MD; Yoshihiro J Akashi ${ }^{1}$, MD

1. Division of Cardiology, Department of Internal Medicine, St. Marianna University School of Medicine

2. Ultrasound Center, St. Marianna University School of Medicine

Address for correspondence:

Shingo Kuwata, MD

Assistant Professor

Division of Cardiology, Department of Internal Medicine, St. Marianna University School of Medicine 2-16-1 Sugao, Miyamae-ku, Kawasaki-city, Kanagawa, Japan

Tel: +81449778111 Fax: +8144976 7093 
E-mail: shingo.k.571019@gmail.com

Disclosure of Potential Conflicts of Interest:

Dr. Kuwata and Izumo is a clinical proctor for Abbott Vascular.

The remaining authors have nothing to disclose.

No financial funding, grants, contracts, and any other forms of financial support to be disclosed for the work of this manuscript.

Word count: 3752 words

\section{Background}

Little is known regarding the impact of right ventricular (RV) function on clinical outcomes following MitraClip therapy.

\section{Objectives}

The aim of this study was to investigate the prognostic impact of RV dysfunction and its cut-off value following MitraClip therapy.

\section{Methods}

Consecutive 77 patients (median 79 years, 33\% female) who underwent MitraClip therapy were enrolled. Clinical endpoint was defined as cardiovascular (CV) events, including cardiovascular death and rehospitalization for heart failure (HF).

\section{Results and conclusions}

Twenty-two (29\%) patients had primary mitral regurgitation (MR). During follow-up, 5 patients died due to CV events, 8 were hospitalized for HF. On univariate Cox regression analysis, CV events were associated with eGFR (HR; 0.960, 95\% CI; 0.926-0.995, $\mathrm{p}=0.027$ ), tricuspid annular plane systolic excursion (TAPSE, HR; 0.874, 95\% CI; 0.789-0.968, p = 0.010), and significant residual MR (HR; 11.652, 95\% CI; 3.257-41.691, $\mathrm{p}<0.001$ ). On multivariate Cox regression analysis, TAPSE (HR; 0.788, 95\% CI; 0.788-0.987, p = 0.029) and significant residual MR (HR; 9.373, 95\% CI; 2.581-34.033, $\mathrm{p}=0.001)$ were independently associated with CV events. TAPSE $<11 \mathrm{~mm}$ was the best cut-off criteria for predicting CV events.

RV function was independently associated with clinical outcomes following MitraClip therapy. TAPSE is a simple parameter for predicting CV events in patients with MR who are undergoing MitraClip therapy.

Key words: MitraClip therapy, mitral regurgitation, right ventricular function, transcatheter mitral valve repair, heart failure, tricuspid annular plane systolic excursion, cardiovascular events

\section{Introduction}

The relationship between right ventricular (RV) function and prognosis has been reported in various cardiac diseases, including mitral regurgitation (MR).(1-6) After transcatheter mitral valve repair (TMVr), patients with RV dysfunction had an increased risk of all-cause mortality.(7) TMVr has the potential to restore RV function, which was shown in early experiences with the MitraClip (Abbott, Abbott Park, IL).(8) To date, only a few reports have provided data regarding RV function during follow-up.(8-10) Further, little is known regarding the impact of RV function and its cut-off value for predicting adverse outcomes following MitraClip therapy. The aim of this study was to investigate the prognostic impact of RV function following TMVr using MitraClip.

\section{Methods}

Patient population 
We included seventy-seven consecutive patients who underwent TMVr using the MitraClip system at a single center in Asia from April 2018 to November 2019. Indications for TMVr included symptomatic, moderateto-severe $(3+)$, or severe $(4+) \mathrm{MR}(11)$ with a high risk for surgery. An interdisciplinary heart team, which included an interventional cardiologist, a cardiac surgeon, an echocardiologist, and a cardiac anesthetist, discussed each subject's eligibility for TMVr. All patients gave written informed consent in a local registry to be included in the study. The protocol of this study was approved by the ethical committee of St. Marianna University School of Medicine.

Transthoracic echocardiographic measurement

Transthoracic echocardiography was performed at baseline (within a week prior to TMVr) and before discharge (shortly after TMVr). MR severity was defined as none or trace $(0 / 4+)$, mild $(1+/ 4+)$, moderate $(2+/ 4+)$, moderate-to-severe $(3+/ 4+)$, and severe $(4+/ 4+)$ using the American Society of Echocardiography (ASE) guidelines for an integrative approach. $(12,13)$ Systolic pulmonary artery (PA) pressure was calculated from the peak tricuspid regurgitant (TR) jet velocity using the simplified Bernoulli's equation, with the addition of the right atrial pressure estimated from inferior vena cava diameter.(14) TR quantification, as well as the evaluation of RV dimensions and function, was performed according to the recommendations of the ASE guidelines.(11,14) Briefly, RV function was assessed through tricuspid annular plane systolic excursion (TAPSE) acquired on M-mode tracings through the tricuspid annulus by the RV-focused apical 4-chamber view (Figure1). RV fractional area change (FAC) by the apical 4-chamber view is the area difference between RV end-diastolic and end-systolic areas measured through ideally RV-focused apical view. RV systolic excursion velocity (S') was defined by tissue Doppler echocardiography as a parameter of the longitudinal velocity of the tricuspid annulus.(15)

\section{Clinical follow-up}

Clinical outcome was defined as the presence or absence of cardiovascular (CV) events, which included cardiovascular death and hospitalization for heart failure (HF). HF was defined as dyspnea and objective signs consistent with New York Heart Association (NYHA) class II-IV requiring hospitalization and medication. Clinical follow-up data were obtained by review of medical records.

Statistical analysis

Data are expressed as median and interquartile range (IQR) for continuous variables and number and percentage for categorical variables. The $t$-test was used to determine between-group differences for continuous variables, and the chi-squared test was used to determine between-group differences for categorical variables. We tested the ability of TAPSE to predict CV events by evaluating the area under the curve (AUC) of its receiver operating characteristic (ROC) curve, and compared its AUC with those of FAC and RV S'. Event-free curves were generated using the Kaplan-Meier method. Log-rank tests were used to evaluate the differences between groups. Multivariate cox regression analysis was used to ascertain the relationship between clinical and echocardiographic variables, which could indicate a potential relationship with outcomes. The results of cox regression analysis are given as hazard ratios (HR) with their respective $95 \%$ confidence intervals (CIs). A probability value of less than 0.05 was considered to indicate statistical significance. Analysis was conducted using a standard statistical software program (SPSS version 19, IBM Corp., Armonk, NY, USA).

\section{Results}

Baseline characteristics are shown in Table 1. The median age of the patients was $79(74-83)$ years, $33 \%$ of patients $(\mathrm{n}=25)$ were female, and the STS SCORE was $11.0(5.3-16.0) \%$. The mechanism of MR was secondary in $55(71 \%)$ patients and primary in $22(29 \%)$ patients. During a median of $221(99-447)$ days of follow-up, $13 \%$ of patients in the entire cohort had CV events (event group). The event group, compared to the no-event group, had lower estimated glomerular filtration rate (eGFR) (25.2 (IQR: 19.9 $31.5) \mathrm{mL} / \mathrm{min} / 1.73 \mathrm{~m}^{2}$ vs. 41.7 (IQR: $\left.29.8-56.6\right) \mathrm{mL} / \mathrm{min} / 1.73 \mathrm{~m}^{2}, \mathrm{p}=0.006$ ), higher N-terminal pro-brain natriuretic peptide (4042 (IQR: $2794-7114) \mathrm{pg} / \mathrm{mL}$ vs. $1760(\mathrm{IQR} ; 870-4955) \mathrm{pg} / \mathrm{mL}, \mathrm{p}=0.039$ ), and lower TAPSE (12 (IQR: $7-14) \mathrm{mm}$ vs. 16 (IQR: $13-22) \mathrm{mm}, \mathrm{p}=0.009$ ). 


\section{Procedural and clinical outcomes}

One clip was implanted in $44(57 \%)$ patients and two clips were implanted in the remaining patients (Table 2). All patients experienced technical success according to the Mitral Valve Academic Research Consortium (MVARC) criteria.(16) During a median of 221 (99-447) days of follow-up after TMVr, 5 patients died due to $\mathrm{CV}$ events and 8 patients had HF hospitalization. The event group, compared to the no-event group, had a higher prevalence of moderate residual MR at discharge $(\mathrm{p}=0.012)$ and longer length of hospital stay postTMVr $(\mathrm{p}=0.003)$ (Table 2). On univariate Cox regression analysis (Table 3), CV events were associated with eGFR (HR: 0.960, 95\%CI; $0.926-0.995, \mathrm{p}=0.027$ ), TAPSE (HR: 0.874, 95\%CI; $0.789-0.968, \mathrm{p}$ $=0.010)$, and significant residual MR (HR: 11.652, 95\% CI; $3.257-41.691, \mathrm{p}<0.001)$. On multivariate Cox regression analysis, CV events were independently associated with TAPSE (HR: 0.788, 95\%CI; 0.788 $-0.987, \mathrm{p}=0.029)$ and significant residual MR (HR: 9.373, 95\%CI; $2.581-34.033, \mathrm{p}=0.001)$. TAPSE did not significantly change mean 4days after the procedure $(15(12-22) \mathrm{mm}$ to $16(12-21) \mathrm{mm}, \mathrm{p}=$ 0.708). Figure 2 shows the ROC analysis of TAPSE to predict CV events. TAPSE had the largest AUC compared to RV-FAC (0.793 vs. 0.681) and RV-S' (0.793 vs. 0.626). TAPSE $<11 \mathrm{~mm}$ was the best cut-off value for predicting CV events (Sensitivity 0.839; Specificity 0.556). Kaplan-Meier analysis showed that no RV functional parameters, except for TAPSE, had a significant prognostic power for predicting CV events (Figure 3).

\section{Discussion}

We aimed to ascertain whether RV dysfunction was associated with CV events in patients with MR who underwent MitraClip therapy. The main findings of this study are as follows: first, RV dysfunction was independently associated with CV events following MitraClip therapy; second, TAPSE was the best RV functional parameter of conventional echocardiography for predicting adverse events; third, the cut-off value of TAPSE for predicting CV events was $11 \mathrm{~mm}$.

RV function is an important parameter with prognostic value in predicting symptomatic limitation and outcome in different cardiovascular pathologies.(17-22) Several parameters have been developed for the evaluation of RV systolic function, including TAPSE, FAC, and S'. Among them, TAPSE is a commonly utilized single-dimension measure of global RV systolic function. It simply measures the distance of systolic excursion of the RV annular segment along its longitudinal axis. According to the ASE/EACVI guidelines, a TAPSE of $<16 \mathrm{~mm}$ suggests impaired RV systolic function.(14) In patients with severe MR, RV dysfunction is associated with increased morbidity and mortality. $(1,23,24)$ Our results are in agreement with those of previous studies $(7,25,26)$ in determining the prognostic role of baseline RV function in patients with MR undergoing MitraClip therapy. Our study expands on these previous studies in demonstrating an 11-mm cut-off value of TAPSE for predicting CV events following MitraClip therapy. Atrial fibrillation (AF) could result in pulmonary hypertension, causing RV dysfunction and RA dilation or loss of atrial contraction, which reduces RV filling and could reduce TAPSE.(27) In the present cohort study, $80 \%$ of the event group had AF. This might have resulted in a low cut-off value for TAPSE.

Apart from RV dysfunction,(28) pulmonary hypertension is a common complication in HF and predicts the occurrence of adverse outcome.(29,30) Pulmonary hypertension has long been considered a serious complication in patients with significant MR. Elevation of LAP occurs in the transitional and decompensated phases of chronic $\mathrm{MR}(31)$ and leads to increased pulmonary arterial pressure, eventually resulting in RV dysfunction.(32)

A number of previous studies have described the impact of RV-PA coupling (TAPSE/PA systolic pressure).(33) Because RV systolic performance is highly dependent on RV afterload,(34) a combination of these coupling parameters would be more important than each parameter in isolation. Combined evaluation (i.e. RV-PA coupling) could be considering right heart hemodynamics.(35) RV-PA coupling ratio was impaired in patients with HF with preserved ejection fraction.(36) Sultan et al. demonstrated that RV-PA coupling is strongly associated with all-cause mortality and its evaluation may be superior to RV or PA alone in predicting worse outcomes in patients undergoing transcatheter aortic valve replacement.(37) In contrast, our 
study demonstrated that TAPSE had a larger AUC than RV-PA coupling (0.793 vs. 0.675) to predict CV events (Supplemental figure 1). On multivariate Cox regression sub-analysis, CV events were independently associated with lower TAPSE (HR: $0.873,95 \% \mathrm{CI} ; 0.788-0.968, \mathrm{p}=0.010$ ) after adjustment for PA pressure measured by transthoracic echocardiography. According to our results, RV dysfunction (by TAPSE) could independently predict worse outcomes regardless of hemodynamics.

In the present study, we found that residual MR $>2+$ was also an independent predictor of CV events. Similarly, a previous study reported that MitraClip therapy was effective in reducing MR.(38,39) Since residual MR after MitraClip therapy has been associated with suboptimal outcomes and increased mortality(40), the goal of the procedure is to reduce residual MR as much as possible.

Limitations

Previous studies demonstrated that RV function improved after MitraClip therapy.(41,42) However, there was no mention of periprocedural change of RV function in this study. This was a single-center study and the sample size was small, which limits its generalizability. Further, this study focused on short-term results. Properly designed trials with longer follow-up and more patients are required to confirm our results.

\section{Conclusions}

TAPSE may be one of the independent significant predictors of CV events in patients with MR undergoing MitraClip therapy. TAPSE may be measured prior to MitraClip therapy as a simple and easy parameter of RV function to predict worse outcomes in a MitraClip cohort.

\section{Clinical Perspectives}

In patients with severe MR, RV dysfunction is associated with increased adverse outcomes. TAPSE was an independent predictor of CV events following MitraClip therapy. Its cut-off value (11\%) may be independently available to predict CV events following MitraClip therapy. The present study comprised a small sample size and focused on short-term results. Properly designed trials with longer follow-up and more patients are required to confirm our results.

\section{Acknowledgments}

The authors thank the structural heart disease Heart Team at St. Marianna University School of Medicine Hospital for their assistance.

References

1. Dini FL, Conti U, Fontanive P et al. Right ventricular dysfunction is a major predictor of outcome in patients with moderate to severe mitral regurgitation and left ventricular dysfunction. Am Heart $\mathrm{J}$ 2007;154:172-9.

2. Merlo M, Gobbo M, Stolfo D et al. The Prognostic Impact of the Evolution of RV Function in Idiopathic DCM. JACC Cardiovasc Imaging 2016;9:1034-1042.

3. Dahou A, Clavel MA, Capoulade R et al. Right ventricular longitudinal strain for risk stratification in low-flow, low-gradient aortic stenosis with low ejection fraction. Heart 2016;102:548-54.

4. Gorter TM, Lexis CP, Hummel YM et al. Right Ventricular Function After Acute Myocardial Infarction Treated With Primary Percutaneous Coronary Intervention (from the Glycometabolic Intervention as Adjunct to Primary Percutaneous Coronary Intervention in ST-Segment Elevation Myocardial Infarction III Trial). Am J Cardiol 2016;118:338-44.

5. Sanz J, Sanchez-Quintana D, Bossone E, Bogaard HJ, Naeije R. Anatomy, Function, and Dysfunction of the Right Ventricle: JACC State-of-the-Art Review. J Am Coll Cardiol 2019;73:1463-1482.

6. Sabe MA, Sabe SA, Kusunose K, Flamm SD, Griffin BP, Kwon DH. Predictors and Prognostic Significance 
of Right Ventricular Ejection Fraction in Patients With Ischemic Cardiomyopathy. Circulation 2016;134:65665.

7. Kaneko H, Neuss M, Weissenborn J, Butter C. Prognostic Significance of Right Ventricular Dysfunction in Patients With Functional Mitral Regurgitation Undergoing MitraClip. Am J Cardiol 2016;118:1717-1722.

8. Giannini C, Petronio AS, De Carlo M et al. Integrated reverse left and right ventricular remodelling after MitraClip implantation in functional mitral regurgitation: an echocardiographic study. Eur Heart J Cardiovasc Imaging 2014;15:95-103.

9. Frangieh AH, Gruner C, Mikulicic F et al. Impact of percutaneous mitral valve repair using the MitraClip system on tricuspid regurgitation. EuroIntervention 2016;11:e1680-6.

10. Vitarelli A, Mangieri E, Capotosto L et al. Assessment of Biventricular Function by Three-Dimensional Speckle-Tracking Echocardiography in Secondary Mitral Regurgitation after Repair with the MitraClip System. J Am Soc Echocardiogr 2015;28:1070-82.

11. Zoghbi WA, Enriquez-Sarano M, Foster E et al. Recommendations for evaluation of the severity of native valvular regurgitation with two-dimensional and Doppler echocardiography. J Am Soc Echocardiogr $2003 ; 16: 777-802$.

12. Zoghbi WA, Adams D, Bonow RO et al. Recommendations for Noninvasive Evaluation of Native Valvular Regurgitation: A Report from the American Society of Echocardiography Developed in Collaboration with the Society for Cardiovascular Magnetic Resonance. J Am Soc Echocardiogr 2017;30:303-371.

13. Lancellotti P, Tribouilloy C, Hagendorff A et al. Recommendations for the echocardiographic assessment of native valvular regurgitation: an executive summary from the European Association of Cardiovascular Imaging. Eur Heart J Cardiovasc Imaging 2013;14:611-44.

14. Rudski LG, Lai WW, Afilalo J et al. Guidelines for the echocardiographic assessment of the right heart in adults: a report from the American Society of Echocardiography endorsed by the European Association of Echocardiography, a registered branch of the European Society of Cardiology, and the Canadian Society of Echocardiography. J Am Soc Echocardiogr 2010;23:685-713; quiz 786-8.

15. Anavekar NS, Gerson D, Skali H, Kwong RY, Yucel EK, Solomon SD. Two-dimensional assessment of right ventricular function: an echocardiographic-MRI correlative study. Echocardiography 2007;24:452-6.

16. Stone GW, Adams DH, Abraham WT et al. Clinical Trial Design Principles and Endpoint Definitions for Transcatheter Mitral Valve Repair and Replacement: Part 2: Endpoint Definitions: A Consensus Document From the Mitral Valve Academic Research Consortium. J Am Coll Cardiol 2015;66:308-321.

17. Aloia E, Cameli M, D'Ascenzi F, Sciaccaluga C, Mondillo S. TAPSE: An old but useful tool in different diseases. Int J Cardiol 2016;225:177-183.

18. Mehta SR, Eikelboom JW, Natarajan MK et al. Impact of right ventricular involvement on mortality and morbidity in patients with inferior myocardial infarction. J Am Coll Cardiol 2001;37:37-43.

19. Polak JF, Holman BL, Wynne J, Colucci WS. Right ventricular ejection fraction: an indicator of increased mortality in patients with congestive heart failure associated with coronary artery disease. J Am Coll Cardiol 1983;2:217-24.

20. Gavazzi A, Berzuini C, Campana $\mathrm{C}$ et al. Value of right ventricular ejection fraction in predicting short-term prognosis of patients with severe chronic heart failure. J Heart Lung Transplant 1997;16:774-85.

21. Shah PK, Maddahi J, Staniloff HM et al. Variable spectrum and prognostic implications of left and right ventricular ejection fractions in patients with and without clinical heart failure after acute myocardial infarction. Am J Cardiol 1986;58:387-93. 
22. Juilliere Y, Barbier G, Feldmann L, Grentzinger A, Danchin N, Cherrier F. Additional predictive value of both left and right ventricular ejection fractions on long-term survival in idiopathic dilated cardiomyopathy. Eur Heart J 1997;18:276-80.

23. Wencker D, Borer JS, Hochreiter C et al. Preoperative predictors of late postoperative outcome among patients with nonischemic mitral regurgitation with 'high risk' descriptors and comparison with unoperated patients. Cardiology 2000;93:37-42.

24. Meluzin J, Spinarova L, Hude P et al. Prognostic importance of various echocardiographic right ventricular functional parameters in patients with symptomatic heart failure. J Am Soc Echocardiogr 2005;18:435-44.

25. Giannini C, Fiorelli F, Colombo A et al. Right ventricular evaluation to improve survival outcome in patients with severe functional mitral regurgitation and advanced heart failure undergoing MitraClip therapy. Int J Cardiol 2016;223:574-580.

26. Orban M, Braun D, Orban M et al. Long-term outcome of patients with severe biventricular heart failure and severe mitral regurgitation after percutaneous edge-to-edge mitral valve repair. J Interv Cardiol 2015;28:164-71.

27. Bazaz R, Edelman K, Gulyasy B, Lopez-Candales A. Evidence of robust coupling of atrioventricular mechanical function of the right side of the heart: insights from M-mode analysis of annular motion. Echocardiography 2008;25:557-61.

28. Mohammed SF, Hussain I, AbouEzzeddine OF et al. Right ventricular function in heart failure with preserved ejection fraction: a community-based study. Circulation 2014;130:2310-20.

29. Bursi F, McNallan SM, Redfield MM et al. Pulmonary pressures and death in heart failure: a community study. J Am Coll Cardiol 2012;59:222-31.

30. Kjaergaard J, Akkan D, Iversen KK et al. Prognostic importance of pulmonary hypertension in patients with heart failure. Am J Cardiol 2007;99:1146-50.

31. Alexopoulos D, Lazzam C, Borrico S, Fiedler L, Ambrose JA. Isolated chronic mitral regurgitation with preserved systolic left ventricular function and severe pulmonary hypertension. J Am Coll Cardiol 1989;14:319-22.

32. Setaro JF, Cleman MW, Remetz MS. The right ventricle in disorders causing pulmonary venous hypertension. Cardiol Clin 1992;10:165-83.

33. Kubba S, Davila CD, Forfia PR. Methods for Evaluating Right Ventricular Function and VentricularArterial Coupling. Prog Cardiovasc Dis 2016;59:42-51.

34. Vonk-Noordegraaf A, Haddad F, Chin KM et al. Right heart adaptation to pulmonary arterial hypertension: physiology and pathobiology. J Am Coll Cardiol 2013;62:D22-33.

35. Cavalcante JL, Simon MA, Chan SY. Comprehensive Right-Sided Assessment for Transcatheter Aortic Valve Replacement Risk Stratification: Time for a Change. J Am Soc Echocardiogr 2017;30:47-51.

36. Hussain I, Mohammed SF, Forfia PR et al. Impaired Right Ventricular-Pulmonary Arterial Coupling and Effect of Sildenafil in Heart Failure With Preserved Ejection Fraction: An Ancillary Analysis From the Phosphodiesterase-5 Inhibition to Improve Clinical Status And Exercise Capacity in Diastolic Heart Failure (RELAX) Trial. Circ Heart Fail 2016;9:e002729.

37. Sultan I, Cardounel A, Abdelkarim I et al. Right ventricle to pulmonary artery coupling in patients undergoing transcatheter aortic valve implantation. Heart 2019;105:117-121.

38. Maisano F, Franzen O, Baldus S et al. Percutaneous mitral valve interventions in the real world: early and 1-year results from the ACCESS-EU, a prospective, multicenter, nonrandomized post-approval study of the MitraClip therapy in Europe. J Am Coll Cardiol 2013;62:1052-1061. 
39. Glower DD, Kar S, Trento A et al. Percutaneous mitral valve repair for mitral regurgitation in high-risk patients: results of the EVEREST II study. J Am Coll Cardiol 2014;64:172-81.

40. Sorajja P, Vemulapalli S, Feldman T et al. Outcomes With Transcatheter Mitral Valve Repair in the United States: An STS/ACC TVT Registry Report. J Am Coll Cardiol 2017;70:2315-2327.

41. Hunlich M, Lubos E, Beuthner BE et al. Acute and Long-Term Hemodynamic Effects of MitraClip Implantation on a Preexisting Secondary Right Heart Failure. Biomed Res Int 2018;2018:6817832.

42. Ledwoch J, Fellner C, Hoppmann P et al. Impact of transcatheter mitral valve repair using MitraClip on right ventricular remodeling. Int J Cardiovasc Imaging 2020.

Table 1. Baseline characteristics

Age, yrs

Female, n (\%)

Body mass index, $\mathrm{kg} / \mathrm{m}^{2}$

Patient history

Previous congestive heart failure, $\mathrm{n}(\%)$

Previous myocardial infarction, $\mathrm{n}(\%)$

Previous coronary artery bypass graft, n (\%)

Previous percutaneous intervention, $\mathrm{n}(\%)$

Previous cerebral disease, n (\%)

Comorbidities

Dyslipidemia, n (\%)

Hypertension, n (\%)

Diabetes mellitus, $\mathrm{n}(\%)$

COPD, n (\%)

Coronary artery disease, $\mathrm{n}(\%)$

Atrial fibrillation, n (\%)

Hemoglobin $(\mathrm{g} / \mathrm{dL})$

eGFR $\left(\mathrm{mL} / \mathrm{min} / 1.73 \mathrm{~m}^{2}\right)$

proBNP $(\mathrm{pg} / \mathrm{mL})$

Patient status

NYHA functional class, n (\%)

I, II, III, IV

STS risk score, $\%$

Preprocedural echo data

Degenerative MR, n (\%)

MR grade; III, IV, n (\%)

MV-EROA, $\mathrm{cm}^{2}$

LVEF, \%

LVEDVi, $\mathrm{mL} / \mathrm{m}^{2}$

LAVi, $\mathrm{mL} / \mathrm{m}^{2}$

RVSP, $\mathrm{mmHg}$

Moderate or severe TR, n (\%)

Pulmonary hypertension, n (\%)

TAPSE, $\mathrm{mm}$

RV-FAC, $\%$

RV-s', cm/s

Values are $\mathrm{n}(\%)$ or median (interquartile range). COPD, chronic obstructive pulmonary disease; eGFR, estimated glomeru 
Table 2. Postprocedural characteristics

Number of clips implanted, n (\%)

Greater to moderate residual MR at discharge, $\mathrm{n}(\%)$

MV mean PG (mmHg)

Length of hospital stay after the procedure (days)

Death, n (\%)

Re-hospitalization due to $\mathrm{HF}, \mathrm{n}(\%)$

Values are $\mathrm{n}(\%)$ or median (interquartile range). HF, heart failure; MR, mitral regurgitation; MV, mitral valve
All patie

1 clip, 44

$5(7)$

$3.0(2.1-$

$7(5-11$

$5(7)$

8 (10)

Table 3. Cox Regression according to cardiovascular death or HF rehospitalization

Variables

NYHA class [?] III

eGFR

Logalism of NT-pro BNP

TR[?]III

TAPSE

Residual MR $>2+$

, Assessed at discharge; , included eGFR, TR III or IV, TAPSE and Residual MR >2+. CI, confidence interval; eGFR, est

Figure Legends

Figure 1.

Title: Transthoracic echocardiographic image of tricuspid annular plane systolic excursion

Legend: Tricuspid annular plane systolic excursion was measured on M-mode tracings through the tricuspid annulus by the RV-focused apical 4-chamber view

$\mathrm{RV}$, right ventricle

Figure 2. (Central Illustration)

Title: ROC curves of TAPSE, RV-FAC, and S' to predict CV events

Legend: The AUC of TAPSE (0.793) was the largest compared to those of RV-FAC (0.681) and S' (0.626).

AUC, area under the curve; CV, cardiovascular; ROC, receiver operating characteristic; TAPSE, tricuspid annular plane systolic excursion

Figure 3.

Title: Kaplan-Meier curves of cardiovascular events according to TAPSE

Legend: Patients with TAPSE $<11 \mathrm{~mm}$ had a significantly higher rate of CV event ( $\mathrm{p}=0.018$ by the log-rank test) compared to those with TAPSE [?]11 mm.

CV, cardiovascular; TAPSE, tricuspid annular plane systolic excursion

Table Legends

Table 1. Baseline characteristics

Table 2. Postprocedural characteristics 
Table 3. Cox Regression according to cardiovascular death or HF rehospitalization
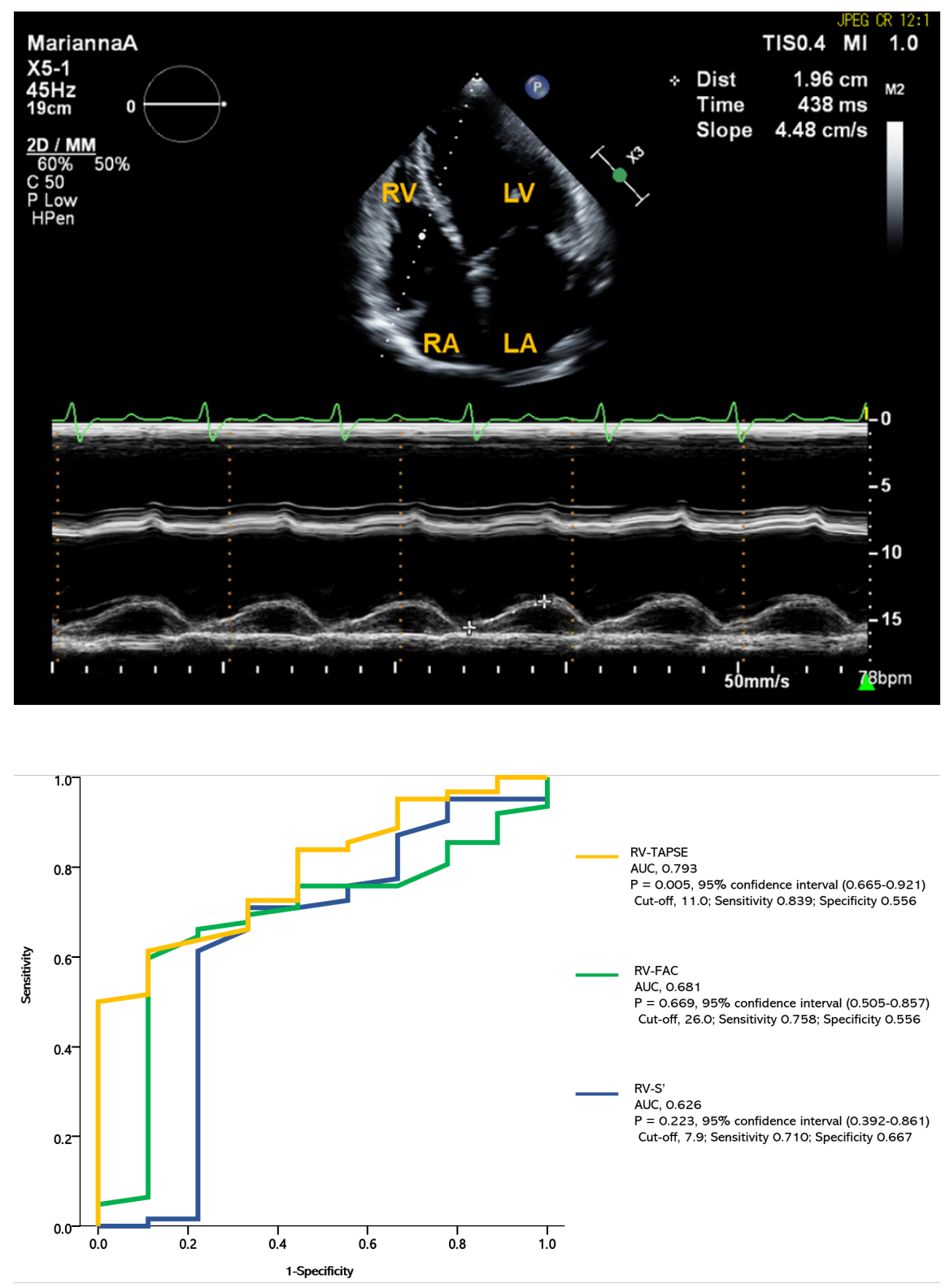


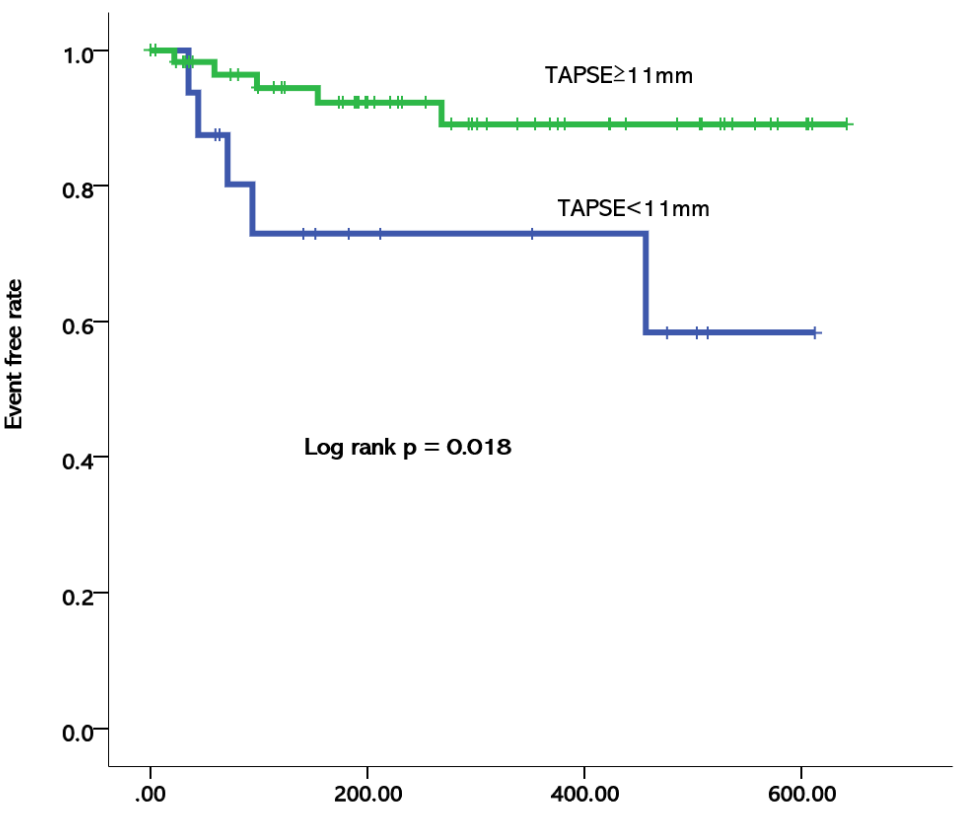

Number at risk

Last follow-up (days)

TAPSE $\geq 11 \mathrm{~mm}$

58

35

17

4

TAPSE $<11 \mathrm{~mm}$

15

7

5

1 